\title{
Semiclassical master equation in Wigners phase space applied to Brownian motion in a periodic potential
}

\author{
W. T. Coffey, ${ }^{1}$ Yu. P. Kalmykov, ${ }^{2}$ S. V. Titov, ${ }^{1,3}$ and B. P. Mulligan ${ }^{1}$ \\ ${ }^{1}$ Department of Electronic and Electrical Engineering, Trinity College, Dublin 2, Ireland \\ ${ }^{2}$ Laboratoire de Mathématiques et Physique des Systèmes, Université de Perpignan, \\ 52, Avenue de Paul Alduy, 66860 Perpignan Cedex, France \\ ${ }^{3}$ Institute of Radio Engineering and Electronics of the Russian Academy of Sciences, \\ Vvedenskii Square 1, Fryazino 141190, Russian Federation
}

(Received 11 December 2006; published 26 April 2007)

\begin{abstract}
The quantum Brownian motion of a particle in a cosine periodic potential $V(x)=-V_{0} \cos \left(x / x_{0}\right)$ is treated using the master equation for the time evolution of the Wigner distribution function $W(x, p, t)$ in phase space $(x, p)$. The dynamic structure factor, escape rate, and jump-length probabilities are evaluated via matrix continued fractions in the manner customarily used for the classical Fokker-Planck equation. The escape rate so yielded is compared with that given analytically by the quantum-mechanical reaction rate solution of the Kramers turnover problem. The matrix continued fraction solution substantially agrees with the analytic solution.
\end{abstract}

DOI: 10.1103/PhysRevE.75.041117

PACS number(s): 05.40.-a, 03.65.Yz, 82.20.-w

\section{INTRODUCTION}

The Brownian motion in a potential is ubiquitous in physics and chemistry, particularly to do with the nature of metastable states and the rates at which these states decay. Typical examples are current-voltage characteristics of Josephson junctions, the rate of condensation of a supersaturated vapor, dielectric and Kerr effect relaxation in liquids and nematic liquid crystals, dynamic light scattering, chemical reaction rate theory in condensed phases, superparamagnetic relaxation, polymer dynamics, nuclear fission and fusion, and so on [1-4]. Now the classical theory of the Brownian motion is well established and is based either on the Langevin equation [1] or on its accompanying Fokker-Planck equation [5]. However, a theory of dissipation based on the classical Brownian motion is often inadequate particularly at low temperatures because it ignores quantum effects. Quantum noise arising from quantum fluctuations is also important in nanoscale and biological systems. We mention [3] the noise assisted tunneling and transfer of electrons and quasiparticles. The characteristics of such quantum noise vary strongly with temperature and at high temperatures a crossover to JohnsonNyquist noise essentially governed by the classical Brownian motion takes place. Yet another aspect of the subject which has come to the fore in recent years is the quantum mechanics of macroscopic quantum variables such as the decay of a zero voltage state in a biased Josephson junction, flux quantum transitions in a superconducting quantum interference device [4] and the possible reversal by quantum tunneling, of the magnetization of a single domain ferromagnetic particle. All these considerations necessitate a theory of quantum Brownian motion particularly one which addresses directly the issue of the quantum-classical correspondence [6] via a quantum analog of the classical Fokker-Planck equation. Such an evolution equation will allow dynamical parameters such as escape rates, correlation times, susceptibilities, etc., to be calculated from the eigensolutions of that equation in a manner analogous to those of the Fokker-Planck equation.
Moreover, it will become possible to compare asymptotic solutions for parameters such as escape rates yielded by reaction rate theory with those calculated from the quantum master equation.

If one wishes to include quantum effects in a diffusion equation treatment, however, a difficulty immediately arises, namely, one cannot speak, because of the uncertainty principle $[7,8]$, of a particle having simultaneously a well defined position and momentum, i.e., the concept of a phase point has no meaning in the quantum world. Therefore, one cannot define as in classical statistical mechanics a probability that the particle has a particular position and a particular momentum. Hence one cannot define a true phase space probability distribution for a quantum mechanical particle. Nevertheless functions bearing some resemblance to phase space distribution functions namely quasiprobability distribution functions have proven [7-11] very useful in quantum mechanical systems as they provide insights into the connection between classical and quantum mechanics allowing one to express quantum mechanical averages in a form which is very similar to that of classical averages. Thus they are ideally suited to the study of the quantum-classical correspondence.

The description of quantum mechanics via phase space distributions advanced by Wigner [7] is an ideal starting point for the formulation of semiclassical quantum master equations. The Wigner phase space formalism $[7,8]$ in quantum mechanics allows one to employ tools of classical physics in the quantum realm. For closed quantum systems, the time behavior of the Wigner function is governed by an evolution equation equivalent to the Schrödinger equation, which in the limit $\hbar \rightarrow 0$ becomes Liouville's equation for the phase space distribution function in classical mechanics. Therefore, the Wigner formalism provides a natural quantum-classical connection.

We should remark that the quantum Brownian motion in a potential may also be treated using many other methods such as numerical simulations [12-15], the reduced density matrix $[16,17]$, path integrals [18], etc. In general, these permit a 
deep understanding of the dynamics of dissipative quantum systems. Moreover, many problems concerning quantum effects on diffusive transport properties, activated barrier crossing, etc., have been solved. However, in spite of the progress achieved these methods possess certain practical disadvantages. For example, a simple time evolution equation for the reduced density matrix does not exist [19]. Moreover, path integrals have been usually confined to harmonic oscillator models since in general it is difficult or indeed impossible to evaluate them for any other potentials [18]. In spite of the formal power of numerical simulation methods, yielding numerically exact solutions, the understanding and interpretation of the qualitative behavior of the relevant physical quantities, is sometimes not at all obvious from them. One would therefore essentially expect that only a combined use of the latter complementary approaches may yield a comprehensive understanding of the quantum dynamics of the Brownian particle in a potential.

Up to the present, little in the nature of detailed solutions of semiclassical master equations for the quantum phase space distribution functions describing quantum Brownian motion in an arbitrary external potential $V(x)$ has appeared in the literature (see, e.g., Refs. [20-22]). Theoretical developments have usually been undertaken for a quantum Brownian harmonic oscillator as treated by Agarwal [23] and others (see, e.g., Refs. [24-27], and references cited therein). However, recently García-Palacios and Zueco [28,29] have proposed an effective method of solving the master equation for the quantum Brownian motion in an anharmonic potential $V(x)$. Their ideas suggest how Brinkman's representation of the classical Fokker-Planck equation as a partial differential recurrence relation in configuration space [30] and its associated solution methods based on matrix continued fractions via a suitable spatial basis for the observables $[1,5]$ could be naturally extended to the quantum regime.

Inspired by these ideas we have recently proposed a quantum master equation for the Brownian motion of a particle in a potential $V(x)$ [31]. Specifically we have demonstrated how the Wigner stationary distribution for closed systems can be used to formally establish a semiclassical master equation allowing one to study the quantum-classical correspondence. Here we show in detail how to solve this master equation for particular quantum systems. In order to illustrate this we consider a particle moving in the periodic potential

$$
V(x)=-V_{0} \cos \left(x / x_{0}\right),
$$

where $x$ is the position of the particle and $x_{0}$ is a characteristic length. Both the classical and quantum Brownian motion in periodic potentials have been used, e.g., to model the diffusion in solids, premelting films, and surfaces (see, for example, Refs. [32-34]). Furthermore, Brownian motion in periodic potentials arises in a number of other important physical applications. We mention the current-voltage characteristics of the Josephson junction [35], mobility of superionic conductors [36], a laser with injected signal [37], phase-locking techniques in radio engineering [38], dielectric relaxation of molecular crystals [39], the dynamics of a charged density wave condensate in an electric field [40], ring-laser gyroscopes [41], stochastic resonance [42,43], etc.
Analytical approaches to the problem are usually based on an ingenious asymptotic method originally devised by Kramers [44] in connection with thermally activated escape of particles from a potential well. His method allows one to determine closed form asymptotic equations for the escape rate in the limits of very low and relatively high dissipation to the heat bath. In this context we must remark that the Kramers escape rate problem in a periodic potential is qualitatively different from that for a metastable well because the periodic potential is multistable. Thus the particle having escaped a particular well may again be trapped due to the thermal fluctuations in another well. Moreover, jumps of either a single lattice spacing or of many lattice spacings are possible. Thus the escape rate in a periodic potential is called the "jump" rate [45]. The Kramers idea was later elaborated upon by Mel'nikov [46] and Mel'nikov and Meshkov [47]. They proposed, based on a Wiener-Hopf equation, a universal formula (that is valid for all values of the dissipation) for the escape rate. Thus they solved the problem of the Kramers turnover between very low and relatively high dissipation, i.e., the calculation of the escape rate $\Gamma$ from a potential well for all values of the damping. Later Grabert [48] and Pollak et al. [49] presented a complete solution of the classical Kramers turnover problem showing that the Mel'nikov turnover formula for the escape rate can be obtained without his ad hoc interpolation between the weak and strong damping regimes. We remark that the theory of Pollak et al. [49] is also applicable to an arbitrary memory friction and not just in the "white noise" (memoryless) limit. A detailed comparison of numerical and analytical approaches to the Brownian motion in the cosine periodic and tilted cosine periodic potentials has been given by Ferrando et al. [45] and Coffey et al. [50]. Moreover, in order to estimate the quantum decay rate for all values of damping, Mel'nikov [46] and Rips and Pollak [51] have further extended the classical method of evaluation of the escape rate $\Gamma$ to account for quantum tunneling in a semiclassical way. By applying this approach to a cosine periodic potential, Georgievskii and Pollak [52] have obtained a universal expression for the quantum rate $\Gamma$ above the crossover temperature between tunneling and thermal activation for the quantum Brownian dynamics in that potential.

Here we solve the semiclassical master equation for the quantum Brownian dynamics in a periodic potential equation (1). In particular we evaluate the dynamic structure factor. This factor allows one to evaluate various physical parameters $[5,45]$. Another of the most important characteristics associated with the Brownian motion in either a single-or a multiwell potential is the friction and temperature dependence of the greatest (overbarrier) relaxation time $\tau$ (or the inverse of the escape rate). The results of exact solutions yielded by the continued fraction method for the damping dependence of $\tau$ will be compared here with those of the Mel'nikov universal equation for the quantum Kramers rate. Such a comparison is yet another purpose of this paper. Thus the validity of the semiclassical approach may be ascertained. 


\section{QUANTUM MASTER EQUATION IN PHASE SPACE}

Wigner [7] showed that quantum mechanics can be reformulated in terms of a phase space $(x, p)$ quasiprobability distribution

$$
W(x, p, t)=\frac{1}{2 \pi \hbar} \int_{-\infty}^{\infty} \rho\left(x+\frac{1}{2} y, x-\frac{1}{2} y\right) e^{-i p y / \hbar} d y,
$$

where $\hbar$ is Planck's constant and $\rho\left(x, x^{\prime}\right)=\left\langle x|\hat{\rho}| x^{\prime}\right\rangle$ is the density matrix. In particular, Wigner's formulation provides a useful tool for introducing quantum corrections to classical models of dissipation such as many body collisions or Brownian motion [25,28,29,53-59]. For quantum Brownian motion, the semiclassical master equation for the translational Brownian motion of a particle in a potential $V(x)$ can be derived by proceeding to the high temperature limit. This is equivalent to treating the system as a quantum mechanical particle embedded in a classical bath [22]. Hence the following semiclassical master equation for the Wigner distribution function $W(x, p, t)$ has intuitive appeal $[25,28,29,57,58]$

$$
\frac{\partial W}{\partial t}+\hat{M}_{W} W=\hat{M}_{D} W
$$

where the operator $\hat{M}_{W}$ is the evolution operator for the closed system (quantum analog of the classical Liouville operator)

$$
\hat{M}_{W} W=\frac{p}{m} \frac{\partial W}{\partial x}-\frac{\partial V}{\partial x} \frac{\partial W}{\partial p}-\sum_{r=1}^{\infty} \frac{(i \hbar / 2)^{2 r}}{(2 r+1) !} \frac{\partial^{2 r+1} V}{\partial x^{2 r+1}} \frac{\partial^{2 r+1} W}{\partial p^{2 r+1}}
$$

and the operator $\hat{M}_{D}$ accounts for effects due to the coupling to the environment (dissipation and fluctuations)

$$
\hat{M}_{D} W=\frac{\partial}{\partial p}\left[D_{p} p W+D_{p p} \frac{\partial W}{\partial p}+D_{x p} \frac{\partial W}{\partial x}\right] \text {. }
$$

Here $D_{p}, D_{p p}$, and $D_{x p}$ are coordinate, momentum, and time dependent parameters which are to be determined. In the classical limit, $\hbar \rightarrow 0$, Eq. (2) reduces as shown, e.g., in Refs. $[24,57]$, to the Klein-Kramers (Fokker-Planck) equation and the coefficients $D_{p}, D_{p p}, D_{x p}$ become

$$
D_{p}=\gamma, \quad D_{p p}=\gamma m / \beta, \quad D_{x p}=0
$$

where $\beta=1 /(k T), k T$ is the thermal energy and $\gamma$ is a dissipation (damping) parameter (measure of the strength of the coupling to the bath). In the quantum case we have evaluated $D_{p}, D_{p p}, D_{x p}$ in the approximation of frequency independent damping [31], where $D_{p}, D_{p p}, D_{x p}$ in Eq. (2) are independent of the time $[25,26]$. In the high temperature limit, this approximation may be used in a wide range of the model parameters both in the limits of weak and strong damping [59]. In this approximation the explicit form of the master equation (2) to $\hbar^{2}$ is [31]

$$
\begin{aligned}
\frac{\partial}{\partial t} W & +\frac{p}{m} \frac{\partial W}{\partial x}-\frac{\partial V}{\partial x} \frac{\partial W}{\partial p}+\frac{\hbar^{2}}{24} \frac{\partial^{3} V}{\partial x^{3}} \frac{\partial^{3} W}{\partial p^{3}}+\cdots \\
& =\gamma \frac{\partial}{\partial p}\left[p W+\frac{m}{\beta}\left\{1+\frac{\hbar^{2} \beta^{2}}{12 m} \frac{\partial^{2} V}{\partial x^{2}}+\cdots\right\} \frac{\partial W}{\partial p}\right] .
\end{aligned}
$$

The main advantage in applying Wigner's phase-space formulation of quantum mechanics to the quantum Brownian motion in a potential is that his formulation proceeds via the master equation (2). This equation is a partial differential equation in phase space akin to the Fokker-Planck equation thus operators are not involved. Furthermore, the phasespace representation suggests how powerful computational techniques developed for the Fokker-Planck equation [5] may be extended to the quantum domain. Using these techniques quantum effects on diffusive transport properties can in principle be estimated for arbitrary potentials. For illustration we consider a particle moving in the periodic potential equation (1). Our present objective is to understand qualitatively how quantum effects treated in semiclassical fashion alter the classical Brownian motion in a periodic potential. Thus now we shall apply matrix continued fractions to calculate various parameters such as the dynamic structure function, the escape rate, etc., directly from Eq. (3) and compare the results so obtained with available analytic solutions.

\section{SOLUTION OF THE MASTER EQUATION (3)}

In order to represent the quantum master equation as a differential recurrence relation for the statistical moments, we make the following rescaling in Eq. (3):

$$
\begin{aligned}
& x^{\prime}=x / x_{0}, \quad p^{\prime}=p \eta /\left(m x_{0}\right), \quad t^{\prime}=t / \eta, \\
& U\left(x^{\prime}\right)=-g \cos x^{\prime}, \quad \Lambda=\beta^{2} \hbar^{2} /\left(48 \eta^{2}\right), \\
& g=\beta V_{0}, \quad \gamma^{\prime}=\eta \gamma, \quad \eta=\sqrt{\beta m x_{0}^{2} / 2} .
\end{aligned}
$$

We then have

$$
\begin{aligned}
\frac{\partial W}{\partial t^{\prime}} & +p^{\prime} \frac{\partial W}{\partial x^{\prime}}-\frac{1}{2} \frac{\partial U}{\partial x^{\prime}} \frac{\partial W}{\partial p^{\prime}}+\frac{\Lambda}{4} \frac{\partial^{3} U}{\partial x^{\prime 3}} \frac{\partial^{3} W}{\partial p^{\prime 3}} \\
& =\frac{\gamma^{\prime}}{2} \frac{\partial}{\partial p^{\prime}}\left[2 p^{\prime} W+\left(1+2 \Lambda \frac{\partial^{2} U}{\partial x^{\prime 2}}\right) \frac{\partial W}{\partial p^{\prime}}\right] .
\end{aligned}
$$

To investigate the process whereby the particle traverses the periodic potential we must obtain the nonperiodic solution of Eq. (4) [5]. Thus we make the ansatz [5]

$$
W\left(x^{\prime}, p^{\prime}, t^{\prime}\right)=\int_{-1 / 2}^{1 / 2} w\left(k, x^{\prime}, p^{\prime}, t^{\prime}\right) e^{-i k x^{\prime}} d k,
$$

where $w$ is periodic in $x^{\prime}$ with period $2 \pi$ and it is assumed that $k$ is restricted to the first Brillouin zone, $-1 / 2 \leqslant k$ $\leqslant 1 / 2$. The periodic function $w$ can then be expanded in a Fourier series in $x$ and in orthogonal Hermite functions $H_{n}\left(p^{\prime}\right)$ in $p^{\prime}[5,45]$, viz., 


$$
w\left(k, x^{\prime}, p^{\prime}, t^{\prime}\right)=\frac{e^{-p^{\prime 2}-U\left(x^{\prime}\right) / 2}}{2 \pi^{3 / 2}} \sum_{n=0}^{\infty} \sum_{q=-\infty}^{\infty} \frac{c_{n, q}\left(k, t^{\prime}\right)}{\sqrt{2^{n} n !}} H_{n}\left(p^{\prime}\right) e^{-i q x^{\prime}} .
$$

By substituting Eq. (6) into Eq. (5), we obtain from Eq. (4) after some algebra that the Fourier coefficients $c_{n, q}\left(k, t^{\prime}\right)$ satisfy the eleven term differential recurrence relation

$$
\begin{aligned}
\frac{d}{d t^{\prime}} c_{n, q} & +\gamma^{\prime}\left[n c_{n, q}-\Lambda g \sqrt{n(n-1)}\left(c_{n-2, q+1}+c_{n-2, q-1}\right)\right] \\
= & i \sqrt{n / 2}\left[(q+k) c_{n-1, q}+g\left(c_{n-1, q+1}-c_{n-1, q-1}\right) / 4\right] \\
& +i \sqrt{(n+1) / 2}\left[(q+k) c_{n+1, q}-g\left(c_{n+1, q+1}-c_{n+1, q-1}\right) / 4\right] \\
& +i \Lambda g \sqrt{n(n-1)(n-2) / 8}\left(c_{n-3, q+1}-c_{n-3, q-1}\right) .
\end{aligned}
$$

By invoking the general method for solving matrix differential-recurrence equations [20,21], we have the solution of Eq. (7) for the spectra $\widetilde{c}_{n, q}(k, \omega)=\int_{0}^{\infty} c_{n, q}(k, t) e^{-i \omega t} d t$ in terms of matrix continued fractions (details of this solution are given in Appendix A).

\section{CALCULATION OF THE OBSERVABLES}

Just as in the classical case, having determined $c_{n, q}(k, t)$, we can evaluate the dynamic structure factor $\widetilde{S}(k, \omega)$ defined as

$$
\tilde{S}(k, \omega)=\int_{0}^{\infty} S(k, t) e^{-i \omega t} d t,
$$

where $S(k, t)=\left\langle e^{i k[x(t)-x(0)]}\right\rangle_{0}$ is the characteristic function of the random variable $x(t)-x(0)$, i.e., the displacement of the particle as it wanders through the wells, and the angular brackets $\langle\cdots\rangle_{0}$ mean equilibrium ensemble averaging. The dynamic structure factor plays a major role in neutron and light scattering experiments [5]. In the present context various physical parameters such as the escape rate, diffusion coefficient, etc., can be evaluated from $\tilde{S}(k, \omega)$. The characteristic function $S\left(k, t^{\prime}\right)$ is calculated in a manner analogous to the classical case $[5,45]$

$$
\begin{aligned}
S\left(k, t^{\prime}\right)= & \left\langle e^{i k\left[x^{\prime}\left(t^{\prime}\right)-x^{\prime}(0)\right]}\right\rangle_{0} \\
= & \int_{-\infty}^{\infty} \int_{-\infty}^{\infty} \int_{-\infty}^{\infty} \int_{-\infty}^{\infty} e^{i k\left(x^{\prime}-x_{0}^{\prime}\right)} \\
& \times W\left(x^{\prime}, p^{\prime}, x_{0}^{\prime}, p_{0}^{\prime}, t\right) d x^{\prime} d x_{0}^{\prime} d p^{\prime} d p_{0}^{\prime} \\
= & \int_{-\infty}^{\infty} \int_{-\infty}^{\infty} \int_{-\infty}^{\infty} \int_{-\infty}^{\infty} e^{i k\left(x^{\prime}-x_{0}^{\prime}\right)} \int_{-1 / 2}^{1 / 2} e^{-i k_{1}\left(x^{\prime}-x_{0}^{\prime}\right)} \\
& \times w\left(k_{1}, x^{\prime}, p^{\prime}, x_{0}^{\prime}, p_{0}^{\prime}, t\right) d k_{1} d x^{\prime} d x_{0}^{\prime} d p^{\prime} d p_{0}^{\prime}
\end{aligned}
$$

[here $x^{\prime}(0)=x_{0}^{\prime}, x^{\prime}(t)=x^{\prime}$ ]. The function $W\left(x^{\prime}, p^{\prime}, x_{0}^{\prime}, p_{0}^{\prime}, t\right)$ $=\int_{-1 / 2}^{1 / 2} e^{-i k\left(x^{\prime}-x_{0}^{\prime}\right)} w\left(k, x^{\prime}, p^{\prime}, x_{0}^{\prime}, p_{0}^{\prime}, t\right) d k$ satisfies Eq. (4) with the initial condition

$$
W\left(x^{\prime}, p^{\prime}, x_{0}^{\prime}, p_{0}^{\prime}, 0\right)=W_{\mathrm{st}}\left(x_{0}^{\prime}, p_{0}^{\prime}\right),
$$

where $W_{\mathrm{st}}\left(x_{0}^{\prime}, p_{0}^{\prime}\right)$ is the equilibrium Wigner distribution function [which is a stationary solution of the master equation (3) [31]]; see Appendix A, Eq. (A5)]. Noting that for a periodic function $f(x)$ and $-1 / 2 \leqslant k, k_{1} \leqslant 1 / 2[5]$

$$
\int_{-\infty}^{\infty} e^{i\left(k-k_{1}\right) x} f(x) d x=\delta\left(k-k_{1}\right) \int_{0}^{2 \pi} f(x) d x,
$$

and utilizing Eq. (6) and the orthogonality properties of the Hermite functions $H_{n}$, the characteristic function $S\left(k, t^{\prime}\right)$ becomes a series of the Fourier coefficients $c_{0, q}\left(k, t^{\prime}\right)$ as [5]

$$
\begin{aligned}
S\left(k, t^{\prime}\right) & =\int_{0}^{2 \pi} \int_{0}^{2 \pi} \int_{-\infty}^{\infty} \int_{-\infty}^{\infty} w\left(k, x^{\prime}, p^{\prime}, x_{0}^{\prime}, p_{0}^{\prime}, t\right) d x^{\prime} d x_{0}^{\prime} d p^{\prime} d p_{0}^{\prime} \\
& =\int_{0}^{2 \pi} \int_{-\infty}^{\infty} w\left(k, x^{\prime}, p^{\prime}, t^{\prime}\right) d x^{\prime} d p^{\prime}=\sum_{q=-\infty}^{\infty} a_{q} c_{0, q}\left(k, t^{\prime}\right),
\end{aligned}
$$

where

$$
a_{q}=(2 \pi)^{-1} \int_{0}^{2 \pi} e^{-i q x-U\left(x^{\prime}\right) / 2} d x^{\prime}
$$

and

$$
w\left(k, x^{\prime}, p^{\prime}, t^{\prime}\right)=\int_{0}^{2 \pi} \int_{-\infty}^{\infty} w\left(k, x^{\prime}, p^{\prime}, x_{0}^{\prime}, p_{0}^{\prime}, t\right) d x_{0}^{\prime} d p_{0}^{\prime} .
$$

Thus the dynamic structure factor $\widetilde{S}(k, \omega)$ then becomes a series of the $\widetilde{c}_{0, q}(k, \omega)$, viz.,

$$
\tilde{S}(k, \omega)=\sum_{q=-\infty}^{\infty} a_{q} \widetilde{c}_{0, q}(k, \omega) .
$$

Thus having calculated $\widetilde{S}(k, \omega)$, we may evaluate the escape (jump) rate $\Gamma$ as follows [45]. The function $S(k, t)$ can be approximated at long times by an exponential

$$
S(k, t)=h(k) e^{-t / \tau(k)} .
$$

The characteristic (longest) relaxation time $\tau(k)$ can then be extracted by representing Eq. (11) in the frequency domain as [45]

$$
\tau(k)=\lim _{\omega \rightarrow 0} \frac{\tilde{S}(k, 0)-\tilde{S}(k, \omega)}{i \omega \tilde{S}(k, \omega)} .
$$

The escape (jump) rate $\Gamma$ is given by

$$
\Gamma \approx 2 \int_{0}^{1 / 2} \tau^{-1}(k) d k
$$

allowing one to estimate the average longest relaxation time of the system since $\tau \sim \Gamma^{-1}$. Now $\tau^{-1}(k)$ can be expressed in terms of the jump rate $\Gamma$ and the jump length probabilities $P_{n}$ (the probability of a jump of length $|n| x_{0} / 2 \pi$ ) as the trigonometric series [45] 


$$
\tau^{-1}(k)=\Gamma \sum_{n=1}^{\infty} P_{n}[1-\cos (2 \pi n k)]
$$

Thus the jump-length probabilities $P_{n}$ may then be obtained in integral form as the Fourier coefficients of the Fourier expansion of $\tau^{-1}(k)$ as detailed in Ref. [45]

$$
P_{n}=-2 \Gamma^{-1} \int_{0}^{1 / 2} \tau^{-1}(k) \cos (2 \pi n k) d k .
$$

For high potential barriers, in the jump diffusion limit, the jump-length probabilities $P_{n}$ allow one to evaluate both the mean-square jump length $\left\langle l^{2}\right\rangle$ and the diffusion coefficient $D$ as [45]

$$
\begin{gathered}
\left\langle l^{2}\right\rangle=4 \pi^{2} x_{0}^{2} \sum_{n=1}^{\infty} n^{2} P_{n}, \\
D \approx(\Gamma / 2)\left\langle l^{2}\right\rangle .
\end{gathered}
$$

The above equations describe in detail the diffusion process in the periodic potential.

\section{MEL'NIKOV'S UNIVERSAL EQUATION}

As already mentioned, Mel'nikov [46] has extended his solution of the classical Kramers turnover problem to include quantum effects in a semiclassical way. He did this by simply inserting the quantum mechanical transmission factor for a parabolic barrier into the classical integral equation for the energy distribution function yielded by the Wiener-Hopf method in the Kramers turnover region. In the approximation of Ohmic damping, he derived a universal formula for the quantum rate $\Gamma^{M}$ valid for all values of damping above the crossover temperature between tunneling and thermal activation

$$
\Gamma^{M}=\Gamma_{\mathrm{IHD}} \Upsilon
$$

Here $\Gamma_{\text {IHD }}$ is the quantum escape rate in the intermediate to high damping (IHD) region $\left(\gamma^{\prime} \geqslant 1\right)$ and $Y$ is the quantum depopulation factor. Furthermore Larkin and Ovchinnikov [60] have generalized Mel'nikov's approach to a system coupled to a bath with Johnson-Nyquist quantum thermal noise spectrum and Mel'nikov and Sütö [61] have applied this method to quantum Brownian motion in a tilted cosine potential [the zero tilt case corresponds to Eq. (1)]. Later Rips and Pollak [51] gave a consistent solution of the quantum Kramers turnover problem demonstrating how the Mel'nikov universal equation (17) can be obtained without his ad hoc interpolation between the weak and strong damping regimes. Finally Georgievskii and Pollak [52] treated the escape rate problem in a periodic cosine potential showing that the quantum depopulation factor $Y$ in Eq. (17) is

$$
Y=4 \int_{0}^{1} \sin ^{2}(\pi k) F(k) d k
$$

The function $F(k)$ is (in our notation)

$$
\begin{aligned}
F(k)= & \exp \left\{\frac{a \sin a}{\pi} \int_{-\infty}^{\infty} \ln \left[\frac{1-e^{-2 R(x)}}{1+e^{-2 R(x)}-2 e^{-R(x)} \cos (2 \pi k)}\right]\right. \\
& \left.\times \frac{d x}{\cosh (2 a x)-\cos a}\right\}, \\
R(x)= & \frac{\pi \gamma^{\prime}}{\sqrt{3 \Lambda}} \int_{-\infty}^{\infty} \frac{\cosh (\sqrt{\Lambda} y)-\cos (2 \sqrt{\Lambda} x y)}{y \sinh (\sqrt{\Lambda} y) \cosh ^{2}[\pi y /(2 \sqrt{6 g})]} d y
\end{aligned}
$$

Here $a=\sqrt{3 \Lambda}\left(\sqrt{\gamma^{\prime 2}+2 g}-\gamma^{\prime}\right)$ and $\delta=8 \gamma^{\prime} \sqrt{2 g}$. If absolute precision is unnecessary the function $R(x)$ from Eq. (20) can be replaced by its classical limit $R(x) \approx \delta\left(x^{2}+1 / 4\right)$. We may now estimate using the Wigner function method the quantum escape rate $\Gamma_{\text {IHD }}$ [and thus $\Gamma^{M}$ via Eq. (17)] by adapting results of the classical Kramers escape rate theory [44] (see Appendix B). The quantum escape rate $\Gamma_{\mathrm{IHD}}$ is then

$$
\Gamma_{\mathrm{IHD}}=\frac{\Xi}{2 \pi \eta}\left(\sqrt{\gamma^{\prime 2}+2 g}-\gamma^{\prime}\right) e^{-2 g},
$$

where

$$
\Xi=\frac{\omega_{c} \sinh \left(\hbar \beta \omega_{a} / 2\right)}{\omega_{a} \sin \left(\hbar \beta \omega_{c} / 2\right)}=1+2 g \Lambda+\cdots
$$

is the quantum correction factor (in full agreement with quantum transition state theory [19]), $\omega_{c}=\sqrt{\left|V^{\prime \prime}\left(x_{c}\right)\right| / m}=\omega_{a}$ $=\sqrt{V^{\prime \prime}\left(x_{a}\right) / m}$. The form of Eq. (21) appears to be consistent with our (Sec. II) conception of a quantum Brownian particle as embedded in a classical bath with the quantum effects in the bath-particle interaction arising via the dependence of the diffusion coefficient on the derivatives of the potential in the quantum master equation. The simple result follows from the exact solution for the Wigner equilibrium distribution function for the harmonic oscillator given in Refs. [8,9].

In the context of solutions of the IHD quantum Kramers rate, we remark that the analysis of Wolynes [62] as well as that of Pollak [63] involves quantization of both bath and particle just as do methods [64] based on Langer's analytical continuation of the free energy. The quantum mechanical enhancement factor $\Xi$ yielded by all these calculations is for Ohmic friction $[19,62]$

$$
\Xi_{W}=\prod_{n=1}^{\infty} \frac{\omega_{a}^{2}+(2 \pi n / \hbar \beta)^{2}+2 \pi n \gamma / \hbar \beta}{-\omega_{c}^{2}+(2 \pi n / \hbar \beta)^{2}+2 \pi n \gamma / \hbar \beta} .
$$

If the condition $\hbar \gamma \beta \ll 2 \pi$ is fulfilled, we have the TST result as $\lim _{\hbar \beta \gamma \rightarrow 0} \Xi_{W}=\Xi[19]$. Thus recovering the result embodied in Eq. (21). The damping independent $\Xi$ is then a fair approximation to $\Xi_{W}$ suggesting that replacement of the equilibrium distribution function by that of the closed system may ultimately yield reasonable semiclassical approximations to the actual time dependent quantum distribution. A comprehensive analysis of Eq. (23) has been made by Hänggi et al. [65] and also by Weiss [19]. They show how the product Eq. (23) may be written as gamma functions 

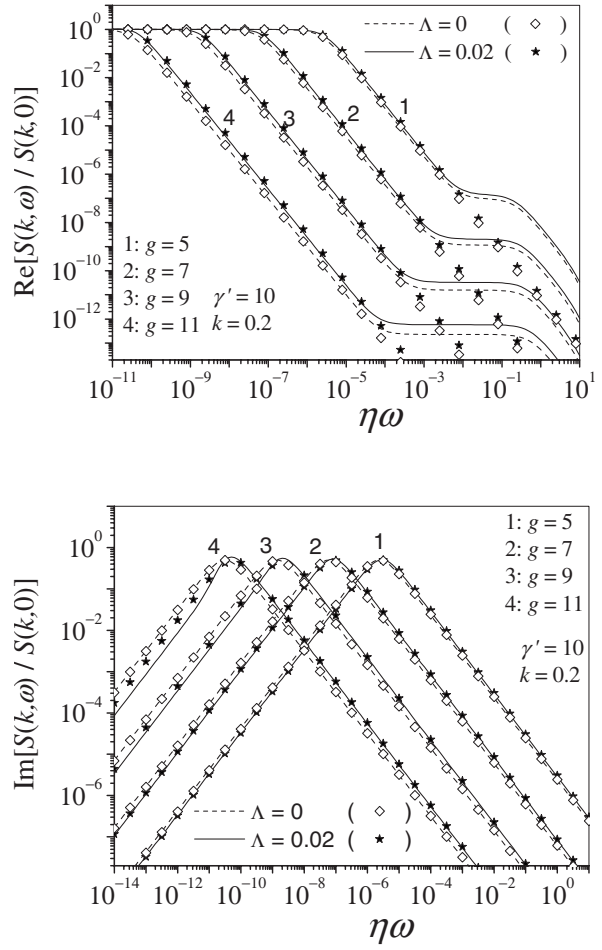

FIG. 1. The real and imaginary parts of the normalized dynamic structure factor $\tilde{S}(k, \omega) / \tilde{S}(k, 0)$ vs $\omega \eta$ for various values of barrier parameter $g=5,7,9$, and 11; the damping coefficient $\gamma^{\prime}=10$ and $k=0.2$. Solid and dashed lines: the matrix continued fraction solution with $\Lambda=0.02$ and $\Lambda=0$ (classical case), respectively. Stars and open diamonds: Eq. (25) with $\Lambda=0.02$ and $\Lambda=0$, respectively.

consequently Wigner's original quantum correction is recovered when $T \gg\left(\gamma / \omega_{c}\right)^{2} T_{c}$. $[45]$

Finally the jump-length probabilities can be estimated as

$$
P_{n}^{M}=-\frac{\int_{0}^{1 / 2} \sin ^{2}(\pi k) F(k) \cos (2 \pi n k) d k}{\int_{0}^{1 / 2} \sin ^{2}(\pi k) F(k) d k},
$$

where $F(k)$ is defined by Eq. (19) and the superscript $M$ denotes analytical calculation (as in Ref. [45]). The results yielded by the analytical theory may now be compared with the matrix continued fraction solution.

\section{RESULTS AND DISCUSSION}

The real and imaginary parts of the normalized dynamic structure factor $\tilde{S}(k, \omega) / \widetilde{S}(k, 0)$ are shown in Fig. 1 for various barrier heights $g$ with the damping parameter $\gamma^{\prime}=10$, and wave number $k=0.2$. For comparison, we also show in this figure the pure Lorentzian spectra

$$
\frac{\widetilde{S}(k, \omega)}{\tilde{S}(k, 0)}=\frac{1}{1+i \omega \tau_{k}},
$$

where the relaxation time $\tau_{k}=\tau_{M}(k)$ is related to the escape $\Gamma^{M}$ from the universal equation (17) via $\Gamma^{M}=2 \int_{0}^{1 / 2} \tau_{M}^{-1}(k) d k$.

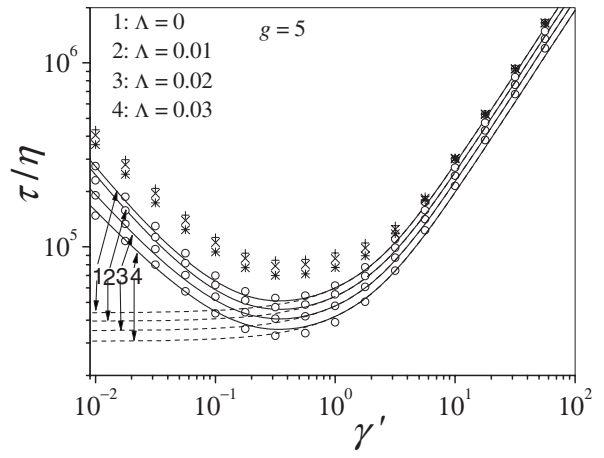

FIG. 2. The normalized longest relaxation time $\tau / \eta$ vs $\gamma^{\prime}$ for the barrier parameter $g=5$ and various values of the quantum parameter $\Lambda=0$ (classical case), 0.01, 0.02, and 0.03 . Solid lines: the universal equation (17). Dashed lines: the IHD equation (21). Open circles: the matrix continued fraction solution of the master equation (4). Symbols: the matrix continued fraction solution of Eq. (4) with the constant diffusion coefficient $D_{p p}=\gamma m / \beta$.

Apparently the simple equation (25) describes perfectly the low frequency part of the normalized dynamic structure factor $\widetilde{S}(k, \omega) / \widetilde{S}(k, 0)$.

The greatest relaxation time $\tau=\Gamma^{-1}$ predicted by the turnover formula Eq. (17) and the inverse decay rate calculated numerically by matrix continued fractions are shown in Fig. 2 as functions of the damping parameter $\gamma^{\prime}$ for various values of the quantum parameters $\Lambda$ (the curves and open circles corresponding to $\Lambda=0$ are the classical results). The IHD [Eq. (21)], asymptotes for $\tau$ are also shown for comparison. Using the Wigner stationary distribution $W_{\mathrm{st}}$ and imposing the condition $\hat{M}_{D} W_{\mathrm{st}}=0$ gives the correct dependence of the escape rate on the quantum parameter $\Lambda(\tau$ decreases with increasing $\Lambda$ ). If the condition $\hat{M}_{D} W_{\text {st }}=0$ is not fulfilled (for example, the diffusion coefficient $D_{p p}$ is regarded as a constant), the behavior of the decay rate is not reproduced at all (see Fig. 2). The quantitative agreement in damping behavior may be explained as follows. The escape rate as a function of the barrier height parameter $g$ for large $g$ is approximately Arrhenius-like and arises from an equilibrium property of the system (namely, the stationary distribution at the bottom of the well). On the other hand the damping dependence of the escape rate is due to nonequilibrium (dynamical) properties of the system so that the Mel'nikov approach [46] should yield the relaxation time for all values of the damping. The greatest relaxation time $\tau$ predicted by the Mel'nikov universal equation (17) and the inverse decay rate calculated numerically via matrix continued fractions are shown in Fig. 3 as functions of $\gamma^{\prime}$ for various barrier heights. The IHD [Eq. (21)] asymptotes for $\tau$ are also shown for comparison. The higher the barrier parameter $g$ the more pronounced is the quantum correction.

The results of calculations of the jump-length probabilities $P_{n}$ and $P_{n}^{M}$ from Eqs. (14) and (24) are shown in Fig. 4 for $\Lambda=0$ (classical case) and $\Lambda=0.02$. The numerical results are consistent with an asymptotic exponential decay of the $P_{n}^{M}$. However, for large $n$ and small friction parameter, $\gamma^{\prime}$ deviations from the exponential behavior may appear [45].

In spite of the very good agreement between the numerical results and the universal equation (17) for $\Lambda<0.03$, a 


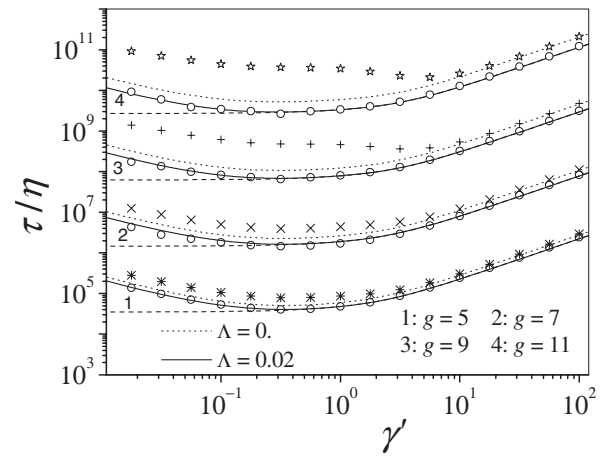

FIG. 3. $\tau / \eta$ vs $\gamma^{\prime}$ for various values of the barrier parameter $g=5,7,9$, and 11. Solid and dotted lines: the universal equation (17) for $\Lambda=0.02$ and $\Lambda=0$ (classical case), respectively. Dashed lines: the IHD equation (21) for $\Lambda=0.02$. Open circles: the matrix continued fraction solution of Eq. (4). Symbols: the matrix continued fraction solution of Eq. (4) with the constant diffusion coefficient $D_{p p}=\gamma m / \beta$.

difference between numerical and analytical results exists in the IHD region for larger values of $\Lambda$. The disagreement indicates that in order to improve the accuracy for these values of $\Lambda$, additional terms of the order of $\Lambda^{2}$, etc. should be included in the master equation. These higher order quantum correction terms to the master equation (3), may be calculated, in principle, to any desired degree $r$ of $\hbar^{2 r}$. However, with increasing $r$, the correction terms become more complicated. In particular, the explicit form of the master equation (2) containing the terms up to $o\left(\hbar^{4}\right)$ is

$$
\begin{aligned}
\frac{\partial W}{\partial t}+ & \frac{p}{m} \frac{\partial W}{\partial x}-\frac{\partial V}{\partial x} \frac{\partial W}{\partial p}+\frac{\hbar^{2}}{24} \frac{\partial^{3} V}{\partial x^{3}} \frac{\partial^{3} W}{\partial p^{3}}-\frac{\hbar^{4}}{1920} \frac{\partial^{5} V}{\partial x^{5}} \frac{\partial^{5} W}{\partial p^{5}}+\cdots \\
= & \gamma \frac{\partial}{\partial p}\left[p W+\frac{m}{\beta}\left\{1+\frac{\hbar^{2} \beta^{2}}{12 m} V^{\prime \prime}-\frac{\hbar^{4} \beta^{4}}{1440 m^{2}}\right.\right. \\
& \left.\left.\times\left[6 V^{\prime \prime \prime} V^{\prime}+2 V^{\prime \prime 2}+3 V^{(4)}\left(\frac{p^{2}}{m}-\frac{5}{\beta}\right)\right]\right\} \frac{\partial W}{\partial p}\right]+\cdots .
\end{aligned}
$$

We emphasize that we use the equilibrium Wigner function $W_{\mathrm{st}}(x, p)$ for vanishing damping $(\gamma \rightarrow 0)$. In quantum systems, however, the equilibrium distribution $W_{\gamma}(x, p)$ is damping dependent [19]. The damping dependence of $W_{\gamma}(x, p)$ is unknown for arbitrary $V(x)$. However, $W_{\gamma}(x, p)$ always reduces to $W_{\mathrm{st}}(x, p)$ in the high temperature limit. Moreover, the difference between $W_{\gamma}(x, p)$ and $W_{\text {st }}(x, p)$ may be negligible in a large range of variation of the model parameters. Thus one would expect that the evolution equation (3) is a reasonable approximation for the kinetics of a quantum Brownian particle in a potential $V(x)$ when $\beta \hbar \gamma \leqslant 1$.

The justification of the master equation (3) for the quantum Brownian motion of a particle in a periodic (cosine) potential (by showing that the solution of that equation for the greatest relaxation time is in agreement with that predicted by quantum rate theory) and the successful extension to the quantum case of the matrix continued fraction methods associated with the classical Fokker-Planck equation are our main results. In particular the dependence of the diffusion

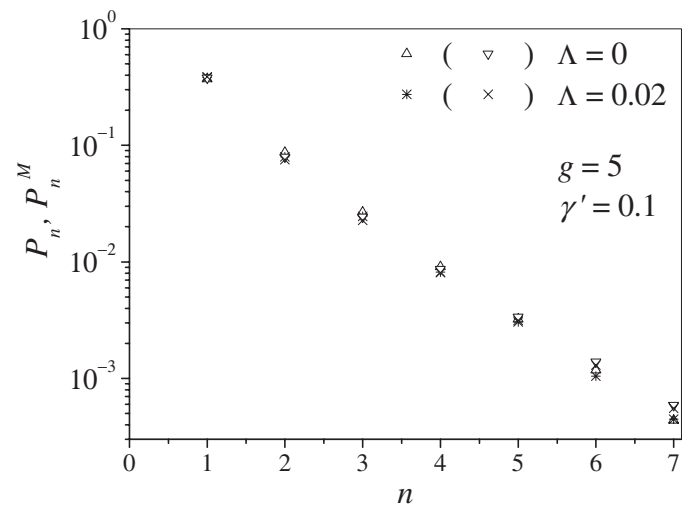

FIG. 4. The jump-length probabilities $P_{n}$ and $P_{n}^{M}$ for the barrier parameter $g=5$, damping parameter $\gamma^{\prime}=0.1$, and two values of the quantum parameter $\Lambda=0$ (up and down triangles; classical case) and $\Lambda=0.02$ (stars and crosses). Up triangles and stars: Eq. (14); down triangles and crosses: Eq. (24).

coefficient on the derivatives of the potential (with consequent lowering of the potential barrier) arising from the ansatz of a Wigner stationary distribution for the equilibrium solution of the open system successfully reproduces escape rates predicted by the quantum generalization of the Kramers escape rate theory and its various extensions to the turnover region as applied to the cosine potential. Furthermore, the successful extension of the classical matrix continued fraction method $[1,5]$ to the semiclassical quantum master equation allows one to accurately calculate in semiclassical fashion quantum corrections to the appropriate dynamical quantities such as correlation functions and susceptibilities (see the calculation of the dynamic structure factor). This is in general impossible using quantum reaction rate theory since that theory as presently formulated does not involve an explicit master equation. We further remark that the agreement obtained between escape rates calculated from quantum reaction rate theory in the manner of Georgievskii and Pollak [52] and those from the master equation (3) (see Figs. 2 and 3 ) also constitutes a verification of quantum rate theory for the potential in question. The above considerations suggest it is obviously worthwhile extending the present study of Eq. (3) to other quantum systems such as the Brownian motion in a periodic potential with tilt, the double-well potential, etc. This will allow one to study the interplay of quantum tunneling, thermal fluctuations, and dissipation in such systems. In particular one will be able to evaluate in semiclassical fashion quantum effects in the spectra of relevant dynamical quantities and the influence of quantum tunneling on the high-temperature behavior of their spectra. We reiterate that the dependence of the diffusion coefficient on the derivatives of the potential arising from the imposition of the Wigner stationary distribution is crucial. If this dependence is not taken into account, e.g., considering the diffusion coefficient as constant, the characteristic lowering of the barrier produced by the quantum tunneling near the top of the barrier cannot be reproduced neither can one regain the results of the quantum reaction rate theory.

Our calculations which have been outlined for mechanical systems with separable and additive Hamiltonians may also 
be extended to particular (nonseparable) spin systems such as a single domain ferromagnetic particle since the giant spin Hamiltonian of the particle may be mapped onto an equivalent single mechanical particle Hamiltonian. This transformation is of particular importance concerning the existence of macroscopic quantum tunneling phenomena in such ferromagnetic particles [66] and also in the discussion of the crossover region between reversal of magnetization by thermal agitation and reversal by macroscopic quantum tunneling which is of current topical interest [67].

\section{ACKNOWLEDGMENTS}

This publication has emanated from research conducted with the financial support of Science Foundation Ireland (Project No. 05/RFP/PHY/0070).

\section{APPENDIX A: MATRIX CONTINUED FRACTION SOLUTION}

First we introduce the column vectors

$$
\mathbf{C}_{n}(t)=\left(\begin{array}{c}
\vdots \\
c_{n-1,-1}\left(k, t^{\prime}\right) \\
c_{n-1,0}\left(k, t^{\prime}\right) \\
c_{n-1,1}\left(k, t^{\prime}\right) \\
\vdots
\end{array}\right) .
$$

Hence the scalar multi-term recurrence equation (7) can be rearranged as the five term matrix differential recurrence relation

$$
\begin{aligned}
\frac{d}{d t} \mathbf{C}_{n}\left(t^{\prime}\right)= & \mathbf{Q}_{n}^{-} \cdot \mathbf{C}_{n-1}\left(t^{\prime}\right)-\gamma^{\prime}(n-1) \mathbf{C}_{n}\left(t^{\prime}\right)+\mathbf{Q}_{n}^{+} \cdot \mathbf{C}_{n+1}\left(t^{\prime}\right) \\
& +\Lambda\left[\mathbf{q}_{n} \cdot \mathbf{C}_{n-2}\left(t^{\prime}\right)+\mathbf{r}_{n} \cdot \mathbf{C}_{n-3}\left(t^{\prime}\right)\right],
\end{aligned}
$$

where the matrix elements of $\mathbf{Q}_{n}^{ \pm}, \mathbf{q}_{n}, \mathbf{r}_{n}$ are given by

$$
\begin{gathered}
\left.\left[\mathbf{Q}_{n}^{ \pm}\right]_{q, p}=i \sqrt{\frac{2 n-1 \pm 1}{4}\left[(q+k) \delta_{q, p} \mp\right.} \frac{g}{4}\left(\delta_{q, p-1}-\delta_{q, p+1}\right)\right], \\
{\left[\mathbf{r}_{n}\right]_{q, p}=i g \sqrt{\frac{(n-1)(n-2)(n-3)}{8}}\left(\delta_{q, p-1}-\delta_{q, p+1}\right),} \\
{\left[\mathbf{q}_{n}\right]_{q, p}=g \gamma^{\prime} \sqrt{(n-1)(n-2)}\left(\delta_{q, p-1}+\delta_{q, p+1}\right),}
\end{gathered}
$$

and $\delta_{q, p}$ is Kronecker's delta. Next we use perturbation theory to find the solution of Eq. (A1) treating $\Lambda$ as the customary small parameter so that we seek a solution as

$$
\mathbf{C}_{n}\left(t^{\prime}\right)=\mathbf{C}_{n}^{0}\left(t^{\prime}\right)+\Lambda \mathbf{C}_{n}^{1}\left(t^{\prime}\right) .
$$

Substituting Eq. (A2) into (A1), we have the matrix threeterm differential recurrence relation for $\mathbf{C}_{n}^{0}\left(t^{\prime}\right)$ in the zeroorder of perturbation theory

$$
\frac{d}{d t^{\prime}} \mathbf{C}_{n}^{0}\left(t^{\prime}\right)=\mathbf{Q}_{n}^{-} \cdot \mathbf{C}_{n-1}^{0}\left(t^{\prime}\right)-\gamma^{\prime}(n-1) \mathbf{C}_{n}^{0}\left(t^{\prime}\right)+\mathbf{Q}_{n}^{+} \cdot \mathbf{C}_{n+1}^{0}\left(t^{\prime}\right)
$$

and in the first order of perturbation theory the forced matrix three-term differential recurrence relation for $\mathbf{C}_{n}^{1}\left(t^{\prime}\right)$, viz.,

$$
\begin{aligned}
\frac{d}{d t^{\prime}} \mathbf{C}_{n}^{1}\left(t^{\prime}\right)= & \mathbf{Q}_{n}^{-} \cdot \mathbf{C}_{n-1}^{1}\left(t^{\prime}\right)-\gamma^{\prime}(n-1) \mathbf{C}_{n}^{1}\left(t^{\prime}\right) \\
& +\mathbf{Q}_{n}^{+} \cdot \mathbf{C}_{n+1}^{1}\left(t^{\prime}\right)+\mathbf{R}_{n}\left(t^{\prime}\right),
\end{aligned}
$$

where $\mathbf{R}_{n}\left(t^{\prime}\right)=\mathbf{q}_{n} \cdot \mathbf{C}_{n-2}^{0}\left(t^{\prime}\right)+\mathbf{r}_{n} \cdot \mathbf{C}_{n-3}^{0}\left(t^{\prime}\right)$.

By invoking the general method $[1,5,28,29]$ for solving three term matrix recursion equations, we have the exact solution for the zero order spectrum $\widetilde{\mathbf{C}}_{n}^{0}(s)=\int_{0}^{\infty} \mathbf{C}_{n}^{0}(t) e^{-s t} d t$ in terms of a matrix continued fraction, viz.,

$$
\widetilde{\mathbf{C}}_{1}^{0}(s)=\boldsymbol{\Delta}_{1}(s) \mathbf{C}_{1}^{0}(0),
$$

$$
\widetilde{\mathbf{C}}_{n}^{0}(s)=\mathbf{S}_{n}^{-} \cdot \mathbf{C}_{n-1}^{0}(s)=\mathbf{S}_{n}^{-} \cdot \mathbf{S}_{n-1}^{-} \cdots \mathbf{S}_{2}^{-} \boldsymbol{\Delta}_{1}(s) \mathbf{C}_{1}^{0}(0),
$$

where $\mathbf{S}_{n}^{-}=\boldsymbol{\Delta}_{n}(s) \mathbf{Q}_{n}^{-}$and the matrix continued fraction $\boldsymbol{\Delta}_{n}(k, s)$ is defined by the recurrence equation

$$
\boldsymbol{\Delta}_{n}(s)=\left\{\left[s+\gamma^{\prime}(n-1)\right] \mathbf{I}-\mathbf{Q}_{n}^{+} \boldsymbol{\Delta}_{n+1}(s) \mathbf{Q}_{n+1}^{-}\right\}^{-1} .
$$

In like manner, we also have the exact solution for the first order spectrum $\widetilde{\mathbf{C}}_{1}^{1}(k, s)$ in terms of a matrix continued fraction, viz.,

$$
\widetilde{\mathbf{C}}_{1}^{1}(s)=\boldsymbol{\Delta}_{1}(s) \mathbf{C}_{1}^{1}(0)+\boldsymbol{\Delta}_{1}(s) \mathbf{S}_{2}^{+} \cdot \mathbf{S}_{3}^{+}\left[\mathbf{C}_{3}^{1}(0)+\mathbf{F}\right],
$$

where $\mathbf{S}_{n}^{+}=\mathbf{Q}_{n-1}^{+} \mathbf{\Delta}_{n}(s)$ and

$\mathbf{F}=\left[\mathbf{q}_{3}+\sum_{n=4}^{\infty} \mathbf{S}_{4}^{+} \cdots \mathbf{S}_{n}^{+}\left(\mathbf{q}_{n} \cdot \mathbf{S}_{n-2}^{-}+\mathbf{r}_{n}\right) \mathbf{S}_{n-3}^{-} \cdots \mathbf{S}_{2}^{-}\right] \boldsymbol{\Delta}_{1}(s) \mathbf{C}_{1}^{0}(0)$.

Here we have noted that $\mathbf{C}_{n}^{0}(0)=\mathbf{0}, n \geqslant 2$ and $\mathbf{C}_{2}^{1}(0)=\mathbf{0}$, $\mathbf{C}_{n}^{1}(0)=\mathbf{0}, n \geqslant 4$.

The initial condition vectors $\mathbf{C}_{n}^{0}(0)$ and $\mathbf{C}_{n}^{1}(0)$ can be calculated just as in the classical case [5] by using the initial condition at $t=0$ for $W\left(x^{\prime}, p^{\prime}, x_{0}^{\prime}, p_{0}^{\prime}, 0\right)=W_{\mathrm{st}}\left(x_{0}^{\prime}, p_{0}^{\prime}\right)$, Eq. (9). However, instead of the Maxwell-Boltzmann distribution of the classical theory, the equilibrium Wigner distribution function $W_{\mathrm{st}}\left(x_{0}^{\prime}, p_{0}^{\prime}\right)$ now has the form [31]

$$
\begin{aligned}
W_{\mathrm{st}}\left(x_{0}^{\prime}, p_{0}^{\prime}\right)= & Z^{-1} e^{-p_{0}^{\prime 2}-U\left(x_{0}^{\prime}\right)}\left\{1+\Lambda\left[U^{\prime 2}\left(x_{0}^{\prime}\right)\right.\right. \\
& \left.\left.+\left(2 p_{0}^{\prime 2}-3\right) U^{\prime \prime}\left(x_{0}^{\prime}\right)\right]\right\},
\end{aligned}
$$

where the partition function $Z$ is given by

$$
\begin{aligned}
Z & =\sqrt{\pi} \int_{0}^{2 \pi}\left\{1+\Lambda\left(U^{\prime}\left(x_{0}^{\prime}\right)\right)^{2}-2 \Lambda U^{\prime \prime}\left(x_{0}^{\prime}\right)\right\} e^{-U\left(x_{0}^{\prime}\right)} d x_{0}^{\prime} \\
& =Z_{\mathrm{cl}}\left[1-\Lambda g I_{1}(g) / I_{0}(g)\right] .
\end{aligned}
$$

Here $Z_{\mathrm{cl}}=2 \pi^{3 / 2} I_{0}(\mathrm{~g})$ is the classical partition function and $I_{0}(x)$ and $I_{1}(x)$ are modified Bessel functions of the first kind. Equations (5), (6), (9), and (A8) yield the initial conditions for $c_{n, q}(t)$ as

$$
c_{n, q}(0)=\frac{1}{\sqrt{2^{n} n !}}\left\langle H_{n}\left(p_{0}^{\prime}\right) e^{i q x_{0}^{\prime}+U\left(x_{0}^{\prime}\right) / 2}\right\rangle_{0},
$$

where the brackets $\langle\cdots\rangle_{0}$ mean the average over $W_{\mathrm{st}}\left(x_{0}^{\prime}, p_{0}^{\prime}\right)$. By representing $c_{n, q}(0)$ via perturbation theory as $c_{n, q}(0)$ $=c_{n, q}^{0}(0)+\Lambda c_{n, q}^{1}(0)$, we have the initial conditions for $c_{n, q}^{0}(0)$ and $c_{n, q}^{1}(0)$, viz., 


$$
\begin{aligned}
& c_{0, q}^{0}(0)= \sqrt{\pi} Z_{\mathrm{cl}}^{-1} \int_{0}^{2 \pi} e^{i q x_{0}^{\prime}-U\left(x_{0}^{\prime}\right) / 2} d x_{0}^{\prime}=2 \pi^{3 / 2} Z_{\mathrm{cl}}^{-1} I_{|q|}(g / 2), \\
& c_{0, q}^{1}(0)= \sqrt{\pi} Z_{\mathrm{cl}}^{-1} \int_{0}^{2 \pi}\left[U^{\prime 2}\left(x_{0}^{\prime}\right)-2 U^{\prime \prime}\left(x_{0}^{\prime}\right)+\frac{g I_{1}(g)}{I_{0}(g)}\right] \\
& \times e^{i q x_{0}^{\prime}-U\left(x_{0}^{\prime}\right) / 2} d x_{0}^{\prime} \\
&=-2 \pi^{3 / 2} Z_{\mathrm{cl}}^{-1}\left[4 q^{2}-g \frac{I_{1}(g)}{I_{0}(g)}\right] I_{|q|}(g / 2), \\
& c_{2, q}^{1}(0)=\sqrt{2 \pi} Z_{\mathrm{cl}}^{-1} \int_{0}^{2 \pi} U^{\prime \prime}\left(x^{\prime}\right) e^{i q x_{0}^{\prime}-U\left(x_{0}^{\prime}\right) / 2} d x_{0}^{\prime} \\
&=\frac{g}{\sqrt{2}}\left[c_{0, q+1}^{0}(0)+c_{0, q-1}^{0}(0)\right] .
\end{aligned}
$$

Having determined $\widetilde{\mathbf{C}}_{1}(k, s)=\widetilde{\mathbf{C}}_{1}^{0}(s)+\Lambda \widetilde{\mathbf{C}}_{1}^{1}(s)$, we can evaluate the dynamic structure factor $\widetilde{S}(k, \omega)$ in terms of $\tilde{c}_{n, q}(k, \omega)$ as

$$
\widetilde{S}(k, \omega)=\frac{Z_{\mathrm{cl}}}{2 \pi^{3 / 2}} \sum_{q=-\infty}^{\infty} c_{0, q}^{0}(0) \widetilde{c}_{0, q}(k, \omega) .
$$

\section{APPENDIX B: ESCAPE RATE IN THE IHD LIMIT}

In order to compare the exact numerical solution with the escape rate obtained from the Kramers theory [44] we adapt the procedure described for the intermediate to high damping classical case in $[68,69]$. In the IHD limit for the cosine potential given by Eq. (1) it is sufficient [46] to consider the escape rate from a single well only. The escape rate considering an isolated well with a source of particles at point $a$ (the bottom of the well) and a potential barrier at point $c$ is then defined by

$$
\Gamma_{\mathrm{IHD}}=\frac{j_{c}}{n_{a}}=\frac{\int_{\text {top }}(p / m) W_{\mathrm{st}}\left(x_{c}, p\right) d p}{\int_{\text {well }} W_{\mathrm{st}}(x, p) d p d x} .
$$

Here $n_{a}$ is the number of particles in the bottom of a potential well at point $a, j_{c}$ is the current across the barrier top at point $c$. The integrals in Eq. (B1) can be estimated via steepest descents by approximating the Wigner functions $W_{\mathrm{st}}\left(x_{c}, p\right)$ and $W_{\mathrm{st}}(x, p)$ [which are the equilibrium solutions of Eq. (4) near the points $a$ and $c$ ] by those of the harmonic oscillator. The number of particles in the well $n_{a}$ is given by

$$
\begin{aligned}
n_{a} & \approx \int_{\text {well }} \int W_{\mathrm{st}}(x, p) d x d p \approx \int_{-\infty}^{\infty} \int_{-\infty}^{\infty} W_{a}(x, p) d x d p \\
& =\frac{\pi \hbar e^{-\beta V\left(x_{a}\right)}}{\sinh \left(\hbar \omega_{a} \beta / 2\right)},
\end{aligned}
$$

where $x_{a}$ is the bottom of the well. Here we have approxi- mated the Wigner function $W_{\mathrm{st}}(x, p)$ [which is the equilibrium solution of Eq. (3) near the point $a]$ by that of the harmonic oscillator, viz. [23],

$$
W_{a}(x, p) \approx \operatorname{sech}\left(\beta \hbar \omega_{a} / 2\right) e^{-\beta V\left(x_{a}\right)-\left(p^{2}+m^{2} \omega_{a}^{2} \bar{x}^{2}\right)\left[\tanh \left(\beta \hbar \omega_{a} / 2\right) / m \hbar \omega_{a}\right]},
$$

where $\omega_{a}=\sqrt{V^{\prime \prime}\left(x_{a}\right) / m}$, and $\bar{x}=x-x_{a}$. In order to calculate the current $j_{c}$ through the barrier at $c$, one needs the Wigner stationary solution near the top (point $c$ ). Here the Wigner function is approximated by that of the inverted harmonic oscillator potential, namely,

$$
W\left(x^{\prime}, p^{\prime}\right)=e^{-\beta V\left(x_{c}\right)} \sec \left(\beta \hbar \omega_{c} / 2\right) e^{-m\left(p^{\prime 2}-\omega_{c}^{2} x^{\prime 2}\right)\left[\tan \left(\beta \hbar \omega_{c} / 2\right) / \hbar \omega_{c}\right]},
$$

where $\omega_{c}=\sqrt{\left|V^{\prime \prime}\left(x_{c}\right)\right| / m}, p^{\prime}=p / m$ and $x^{\prime}=x-x_{c}$. Furthermore, near the top we have from Eq. (3)

$$
p^{\prime} \frac{\partial W_{c}}{\partial x^{\prime}}+\omega_{c}^{2} x^{\prime} \frac{\partial W_{c}}{\partial p^{\prime}}=\frac{\partial}{\partial p^{\prime}}\left[\gamma p^{\prime} W_{c}+D_{p^{\prime} p^{\prime}} \frac{\partial W_{c}}{\partial p^{\prime}}\right],
$$

where $D_{p^{\prime} p^{\prime}}=\left(\gamma \hbar \omega_{c} / 2 m\right) \cot \left(\beta \hbar \omega_{c} / 2\right)$. Equation (B3) has the form of a Boltzmann distribution and satisfies Eq. (B4). This fact allows us to write following Kramers $[44,65]$ the nonequilibrium solution $W\left(x^{\prime}, p^{\prime}\right)$ near the barrier as

$$
W_{c}\left(x^{\prime}, p^{\prime}\right)=C F\left(x^{\prime}, p^{\prime}\right) e^{-\beta^{\prime}\left(p^{\prime 2}-\omega_{c}^{2} x^{\prime 2}\right)},
$$

where $C=e^{-\beta V\left(x_{c}\right)} \sec \left(\hbar \omega_{c} \beta / 2\right)$ and $\beta^{\prime}=m \tan \left(\hbar \omega_{c} \beta / 2\right) /$ $\left(\hbar \omega_{c}\right)$. The function $F\left(x^{\prime}, p^{\prime}\right)$ is a crossover function which has the equilibrium distribution in the depths of the well, varies very rapidly in the vicinity of the barrier and vanishes beyond the barrier as in the classical Kramers case $[44,66]$. Consequently that function must satisfy the boundary conditions

$$
F\left(x^{\prime}, p^{\prime}\right) \rightarrow \begin{cases}1, & x^{\prime} \rightarrow \infty, \\ 0, & x^{\prime} \rightarrow-\infty .\end{cases}
$$

By substituting Eq. (B5) into Eq. (B4) and noting that $D_{p^{\prime} p^{\prime}} \beta^{\prime}=\gamma / 2$, we have the differential equation for the crossover function as in the classical case $[44,64,65]$

$$
p^{\prime} \frac{\partial F}{\partial x^{\prime}}+\omega_{c}^{2} x^{\prime} \frac{\partial F}{\partial p^{\prime}}=D_{p^{\prime} p^{\prime}} \frac{\partial^{2} F}{\partial p^{\prime 2}}-\gamma p^{\prime} \frac{\partial F}{\partial p^{\prime}} .
$$

The solution of Eq. (B7) is of the form $F\left(x^{\prime}, p^{\prime}\right)=F\left(p^{\prime}\right.$ $\left.-a x^{\prime}\right)$. By substituting $F\left(p^{\prime}-a x^{\prime}\right)$ into Eq. (B7) and introducing a new variable $\xi=p^{\prime}-a x$, we have

$$
\left[(a-\gamma) p^{\prime}-\omega_{c}^{2} x^{\prime}\right] \frac{\partial F}{\partial \xi}+D_{p^{\prime} p^{\prime}} \frac{\partial^{2} F}{\partial \xi^{2}}=0 .
$$

Equation (B8) simplifies to an ordinary differential equation if $\omega_{c}^{2}=(a-\gamma) a$ or

$$
a-\gamma=\sqrt{\gamma^{2} / 4+\omega_{c}^{2}}-\gamma / 2
$$

(this is the condition that the eigenvalue associated with the unstable barrier crossing mode is real), namely,

$$
(a-\gamma) \xi \frac{\partial F}{\partial \xi}+D_{p^{\prime} p^{\prime}} \frac{\partial^{2} F}{\partial \xi^{2}}=0 .
$$


The solution of Eq. (B9) satisfying the boundary conditions Eq. (B6) is

$$
F(\xi)=\frac{1}{\sqrt{\pi}} \int_{-\infty}^{\xi \sqrt{(a-\gamma) / 2 D_{p^{\prime} p^{\prime}}}} e^{-y^{2}} d y
$$

The current $j_{c}$ is then given by

$$
\begin{aligned}
j_{c} & =m \int_{-\infty}^{\infty} p^{\prime} W_{c}\left(0, p^{\prime}\right) d p^{\prime} \\
& =\frac{m C}{\sqrt{\pi}} \int_{-\infty}^{\infty} p^{\prime} e^{-\beta^{\prime} p^{\prime 2}} \int_{-\infty}^{p^{\prime} \sqrt{(a-\gamma) / 2 D_{p^{\prime} p^{\prime}}}} e^{-y^{2}} d y d p^{\prime} \\
& =\frac{\hbar e^{-\beta V\left(x_{c}\right)}}{2 \sin \left(\hbar \beta \omega_{c} / 2\right)}\left(\sqrt{\gamma^{2} / 4+\omega_{c}^{2}}-\gamma / 2\right) .
\end{aligned}
$$

Substituting Eqs. (B2) and (B11) into Eq. (B1) then yields the escape rate $\Gamma_{\mathrm{IHD}}$.
[1] W. T. Coffey, Yu. P. Kalmykov, and J. T. Waldron, The Langevin Equation, 2nd ed. (World Scientific, Singapore, 2004).

[2] J. S. Langer, Ann. Phys. (N.Y.) 54, 258 (1969).

[3] P. Hänggi and G. L. Ingold, Chaos 15, 026105 (2005).

[4] P. Hänggi, P. Talkner, and M. Berkovec, Rev. Mod. Phys. 62, 251 (1990).

[5] H. Risken, The Fokker-Planck Equation, 2nd ed. (Springer, Berlin, 1989).

[6] D. Kohen and D. J. Tannor, Adv. Chem. Phys. 111, 219 (1994).

[7] E. P. Wigner, Phys. Rev. 40, 749 (1932).

[8] M. Hillery, R. F. O'Connell, M. O. Scully, and E. P. Wigner, Phys. Rep. 106, 121 (1984); V. I. Tatarskii, Usp. Fiz. Nauk 139, 587 (1983) [Sov. Phys. Usp. 26, 311 (1983)].

[9] H. W. Lee, Phys. Rep. 259, 147 (1995).

[10] W. P. Schleich, Quantum Optics in Phase Space (Wiley-VCH, Berlin, 2001).

[11] R. Kapral, Annu. Rev. Phys. Chem. 57, 129 (2006).

[12] M. Topaler and N. Makri, J. Chem. Phys. 101, 7500 (1994).

[13] N. Makri, J. Math. Phys. 36, 2430 (1995).

[14] C. H. Mac and R. Egger, Adv. Chem. Phys. 93, 39 (1995).

[15] E. Pollak, J. Bader, B. J. Berne, and P. Talkner, Phys. Rev. Lett. 70, 3299 (1993).

[16] A. G. Redfield, IBM J. Res. Dev. 1, 19 (1957).

[17] Y. J. Yan, F. Shuang, R. Xu, J. Cheng, X. Q. Li, C. Yang, and H. Zhang, J. Chem. Phys. 113, 2068 (2000).

[18] H. Grabert, P. Schramm, and G. L. Ingold, Phys. Rep. 168, 115 (1988).

[19] U. Weiss, Quantum Dissipative Systems (World Scientific, Singapore, 1999).

[20] H. T. Davis, K. Hiroike, and S. A. Rice, J. Chem. Phys. 43, 2633 (1965).

[21] Y. Tanimura and P. G. Wolynes, J. Chem. Phys. 96, 8485 (1992).

[22] S. Zhang and E. Pollak, J. Chem. Phys. 118, 4357 (2003).

[23] G. S. Agarwal, Phys. Rev. A 4, 739 (1971).

[24] H. Dekker, Phys. Rev. A 16, 2116 (1977).

[25] J. J. Halliwell and T. Yu, Phys. Rev. D 53, 2012 (1996).

[26] R. Karrlein and H. Grabert, Phys. Rev. E 55, 153 (1997).

[27] G. W. Ford and R. F. O'Connell, Phys. Rev. D 64, 105020 (2001).

[28] J. L. García-Palacios and D. Zueco, J. Phys. A 37, 10735 (2004).

[29] J. L. García-Palacios, Europhys. Lett. 65, 735 (2004).
[30] H. C. Brinkman, Physica (Amsterdam) 22, 29 (1956).

[31] W. T. Coffey, Yu. P. Kalmykov, S. V. Titov, and B. P. Mulligan, Europhys. Lett. 77, 20011 (2007); J. Phys. A 40, F91 (2007).

[32] E. Pollak, J. Bader, B. J. Berne, and P. Talkner, Phys. Rev. Lett. 70, 3299 (1993).

[33] R. Ferrando, R. Spadacini, and G. E. Tommei, Nuovo Cimento D 15, 557 (1993).

[34] S. Miret-Artés and E. Pollak, J. Phys.: Condens. Matter 17, S4133 (2005).

[35] A. Barone and G. Paterno, Physics and Applications of the Josephson Effect (Wiley, New York, 1982).

[36] W. Dieterich, P. Fulde, and I. Peschel, Adv. Phys. 29, 527 (1980).

[37] W. W. Chow, M. O. Scully, and E. W. Van Stryland, Opt. Commun. 15, 6 (1975).

[38] A. J. Viterbi, Principles of Coherent Communication (McGraw-Hill, New York, 1966).

[39] W. T. Coffey, Adv. Chem. Phys. 63, 69 (1985).

[40] S. G. Chung, Phys. Rev. B 29, 6977 (1984).

[41] W. W. Chow, J. Gea-Banacloche, L. M. Pedrotti, V. Sanders, W. Schleich, and M. O. Scully, Rev. Mod. Phys. 57, 61 (1985).

[42] L. Gammaitoni, P. Hänggi, P. Jung, and F. Marchesoni, Rev. Mod. Phys. 70, 223 (1998).

[43] I. Kh. Kaufman, D. G. Luchinsky, P. V. E. McClintock, S. M. Soskin, and N. D. Stein, Phys. Rev. E 57, 78 (1998).

[44] H. A. Kramers, Physica (Amsterdam) 7, 284 (1940).

[45] R. Ferrando, R. Spadacini, and G. E. Tommei, Phys. Rev. E 48, 2437 (1993).

[46] V. I. Mel'nikov, Phys. Rep. 209, 1 (1991); Physica A 130, 606 (1985).

[47] V. I. Mel'nikov and S. V. Meshkov, J. Chem. Phys. 85, 1018 (1986).

[48] H. Grabert, Phys. Rev. Lett. 61, 1683 (1988).

[49] E. Pollak, H. Grabert, and P. Hänggi, J. Chem. Phys. 91, 4073 (1989).

[50] W. T. Coffey, Yu. P. Kalmykov, S. V. Titov, and B. P. Mulligan, Phys. Rev. E 73, 061101 (2006).

[51] I. Rips and E. Pollak, Phys. Rev. A 41, 5366 (1990).

[52] Yu. Georgievskii and E. Pollak, Phys. Rev. E 49, 5098 (1994).

[53] C. W. Gardiner and P. Zoller, Quantum Noise, 2nd ed. (Springer, Berlin, 2000).

[54] M. Ploszajczak and M. J. Rhoades-Brown, Phys. Rev. Lett. 
55, 147 (1985).

[55] D. Cohen, Phys. Rev. Lett. 78, 2878 (1997).

[56] L. M. Johansen, Phys. Rev. Lett. 80, 5461 (1998).

[57] A. O. Caldeira and A. J. Leggett, Physica A 121, 587 (1983).

[58] Y. Tanimura and P. G. Wolynes, Phys. Rev. A 43, 4131 (1991).

[59] H. Grabert, Chem. Phys. 322, 160 (2006).

[60] A. I. Larkin and Yu. N. Ovchinnikov, J. Stat. Phys. 41, 425 (1985).

[61] V. I. Mel'nikov and A. Sütö, Phys. Rev. B 34, 1514 (1986).

[62] P. G. Wolynes, Phys. Rev. Lett. 47, 968 (1981).

[63] E. Pollak, Chem. Phys. Lett. 127, 178 (1986); E. Pollak, J. Chem. Phys. 85, 865 (1986)
[64] V. A. Benderskii, D. E. Makarov, and C. A. Wright, Adv. Chem. Phys. 88, 1 (1994).

[65] P. Hänggi, H. Grabert, G. L. Ingold, and U. Weiss, Phys. Rev. Lett. 55, 761 (1985).

[66] C. P. Bean and J. D. Livingston, J. Appl. Phys. 30, 120 S (1959).

[67] W. Wernsdorfer, Adv. Chem. Phys. 118, 99 (2001).

[68] Selected Papers on Noise and Stochastic Processes, edited by N. Wax (Dover, New York, 1954).

[69] W. T. Coffey, D. A. Garanin, and D. J. McCarthy, Adv. Chem. Phys. 117, 528 (2001). 